\section{QUASARS AND PULSARS}

\section{The Sume Inside?}

IT had to happen. In this month's Astrophysical Journal Letters (15\%, L73; 1969) Professor Philip Morrison of MIT asks "Are quasi-stellar radio sources giant pulsars ?" and goes on to list a handful of features common to both. Quasars, for example, can be periodic. Their radiation is polarized, indicating magnetic fields in the source. They are highly concentrated objects. And, like pulsars, they could easily be the remnants of explosions. Their evolution, of course, is different, but Morrison is saying that, apart from differences of scale, they are objects of the same kind. What they have in common is a spinning, magnetized core of condensed material, and in both cases the work done by the spinning magnetic field on charged particles emitted from the core is an important cause of slowdown.

Morrison's prototype quasar is $3 C$ 345, which has a sharp optical outburst at intervals of $320+3$ days. According to Morrison, this is a reasonable value for the rotation period of a condensed galactic core. Naturally enough, his typical pulsar is the Crab pulsar, $N P 0532$, and its history as the stellar remnant of a supernova suggests how quasars might have evolved.

Like almost everybody these days, Morrison sees quasars as belonging to the same family tree as normal galaxies, Seyfert galaxies and so on. What happens, he suggests, is the contraction of the nucleus of a normal galaxy into a rapidly spinning, intensely magnetized core. Interaction of the spinning field with charged particles, presumably from a hot-spot on the core, is responsible for the bursts of radiation.

And, just as NP 0532 is immersed in the Crab nebula, quasars on this model are surrounded by a cloud of gas which is excited by the supply of energetic particles from the core. It is the gas in the near neighbourhood of the central core which is responsible for the optical continuum, while the bulk of the radio power comes from the more remote parts of the cloud-the analogue of the extended filaments of the Crab. Just as in the Crab, Morrison says, the periodic radio pulses are likely to be much weaker than the optical pulses and swamped by the radio emission from the outer regions. This is why, he says, no radio bursts have so far been detected to match the optical pulsations.

Morrison sums up his paper in a nutshell: quasistellar objects are to quasi-stellar sources as the radio pulsars are to the Crab pulsar itself-ageing objects which have lost their accompanying clouds of excited gas. In other words, they are galactic coffins.

\section{MOON}

\section{Next Penumbral Eclipse}

THE last eclipse of the year will occur on September 25, when the Moon will pass through the penumbra of the Earth's shadow. The maximum of the eclipse will be at 20.10 GMT, when 93 per cent of the Moon will be covered by the shadow. According to The American Ephemeris and Nautical Almanac, the beginning of the eclipse at 18.06 will be visible in Asia, the western Pacific Ocean, Australia, New Zealand, the Indian Ocean, Africa except the north-western part, Europe except the western part, and the Arctic regions. The ending at 22.15 will be visible in Asia except the eastern part, the Indian Ocean, Africa, Europe, the Atlantic Ocean, South America except the western part, the extreme north-eastern part of North America, and the Arctic regions.

Although a lunar eclipse has little of the importance of a solar eclipse, scientific interest has been aroused over the question of contributions to the luminosity of the Moon when the solar illumination is cut off. In the case of a penumbral eclipse, the Earth's atmosphere has the effect of slightly increasing the angular radius of the Earth as viewed from the Moon, and thus increasing the density of the penumbra. On the other hand, refraction in the atmosphere tends to brighten the shadow, and on balance the refraction effect prob. ably dominates (Link, F., Eclipse Phenomena, SpringerVerlag, 1969).

But observations seem to show an excess of light in the part of the penumbra nearest the geometrical shadow and a deficiency in the outer regions. Neither scattered light from the atmosphere nor the influence of the structure of lunar soil could be responsible. The answer seems to be the luminescence of lunar material, and the variation of the light excess across the penumbra could be due to the way the Earth, seen from different parts of the Moon, blocks off sources of radiation on the Sun.

The two solar eclipses of 1969 have been disappointing for astronomers-both have been in remote parts of the world and, worse, both have been annular eclipses. This means that the apparent diameter of the Moon is appreciably smaller than that of the Sun so that an annulus of the Sun is still visible even at maximum. This happens when the Moon happens to be at the apogee of its orbit around the Earth-the apogee and perigee differ by about $50,000 \mathrm{~km}$.

But next year a total solar eclipse on March 7 will start in the Pacific Ocean, cross Central America and sweep up the eastern seaboard of the United States and Canada. Expeditions are already being planned.

\section{PARTICLE PHYSICS}

\section{Quarks or Red Herrings?}

THERE has been a mixed reaction to the announcement last week by Professor C. B. McCusker of the University of Sydney that his group has found evidence for the existence of fractionally charged particles in a new type of cosmic ray experiment. Among high-energy physicists, there is both scepticism and muted optimism. Speaking to the international cosmic ray conference in Budapest, Professor McCusker reported the results of an experiment in which cosmic ray showers from primary radiation, with energy about $10^{15} \mathrm{eV}$, have been analysed with a set of cloud chambers. He said that, of the 60,000 tracks obtained in the experiment, there were five that could be explained by the existence of a particle with a charge two-thirds that of the proton. This is the charge expected for one of the three brands of quarks.

The experiment itself was basically straightforward. The centre or core region of a shower of secondary cosmic ray particles was selected by a core detector, and four cylindrical cloud chambers were used to obtain ionization tracks from the shower. In all, 